\title{
Rhéologie des vases molles
}

\author{
Khosrow Hosseini \\ Chercheur, LMG, Faculté des Sciences et des Techniques, Université de Nantes \\ Martin Sanchez \\ Maitre de conférences, LMG, Université de Nantes \\ Alain Grovel \\ Professeur des Universités, $L M G$, Université de Nantes
}

\section{Résumé}

L'étude rhéologique des sédiments cohésifs permet de déterminer des paramètres importants pour préciser leur comportement sédimentaire sous une action hydrodynamique. L'un des paramètres définissant le meux le caractère liquide, plastique ou solide, d'une vase, est la rigidité initiale.

Dans cette étude on a mis au point une démarche pour mesurer la rigidité initiale en utilisant un densimètre classique. Les mesures s'avèrent répétitives et comportent une incertitude relative faible qui peut être chiffrée.

La mesure en parallèle de la rigidité initiale au rhéomètre montre qu'il existe une proportionnalité entre celle-ci et celle mesurée grâce au densimètre.

Enfin, l'utilisation d'une plaque inclinée permet d'observer qualitativement l'écoulement ou la déformation de la vase.

\section{Introduction}

Suivant les valeurs de la concentration, une vase suit des lois théologiques différentes. Lorsque la concentration d'une mélange eau-vase est inférieure à une certaine valeur, elle se comporte comme un fluide newtonien ou pseudoplastique ; au-delà de cette concentration, la vase se comporte comme un fluide viscoplastique ayant une rigidité initiale, laquelle est une fonction croissante de la concentration. Usuellement, l'étude du comportement des fluides et des matériaux viscoplastiques se fait à l'aide d'un rhéomètre en mesurant le couple nécessaire pour imprimer une vitesse rotation donnée à un cylindre immergé dans le matériau étudié.

\subsection{Comportement rhéologique de la crème de vase}

Pour des concentrations supérieures à une valeur critique la crème de vase a un comportement viscoplastique, c'est-à-dire, qu'il n'y a pas d'écoulement tant que la contrainte est inférieure à une certaine valeur $\tau_{\mathrm{y}}$ qui représente la rigidité initiale. Au-delà de ce seuil de plasticité, le comportement de la vase peut être linéaire (modèle de Bingham) ou non linéaire. 


$$
\tau=\tau_{B}+\mu_{B} \cdot \frac{d u}{d y} \quad \text { (modèle de Bingham) }
$$

où $\tau$ est la tension de cisaillement $[\mathrm{Pa}], \tau_{B}$ le seuil de plasticité $[\mathrm{Pa}], \mu_{B}$ la viscosité dynamique $[\mathrm{Pa} s]$, l'indice $\mathrm{B}$ réfère à Bingham et du/dy est la vitesse de déformation ou gradient de vitesse[ $\left[\mathrm{s}^{-1}\right]$.

Certaines vases ont un caractère thixotropique : leur viscosité diminue avec un certain retard sous l'effet de l'agitation. La thixotropie est observée grâce aux rhéogrammes où l'on représente la tension de cisaillement en fonction de la vitesse de déformation pour des valeurs croissantes et décroissantes de du/dy ; l'ampleur de la boucle augmente avec l'importance de la thixotropie [5].

Pour mesurer la rigidité initiale de la vase on utilise couramment les viscosimètres à cylindres. Le principe est de mesurer le moment de torsion nécessaire pour mettre en mouvement un cylindre immergé dans le matériau.

Grâce aux mesures réalisées au LCHF à l'aide d'un viscosimètre Brookfield LVT sur des vases diverses, Migniot [3] a observé une relation entre la rigidité initiale et la concentration $\mathrm{C}$ comme suite :

$$
\tau_{y}=b C^{a}
$$

où $\tau_{y} \mathrm{~s}^{\prime}$ exprime en $\mathrm{Pa}$ et $\mathrm{C}$ en $\mathrm{kg} \mathrm{m}^{-3}$; a et $\mathrm{b}$ sont des coefficients propres à chaque vase. Pour la vase plastique de la Loire, il a évalué les coefficients suivants:

$$
a=6 \text { et } b=21 \times 10^{-15}
$$

La rigidité initiale est un paramètre largement utilisé pour caractériser les sédiments cohésifs. Certains auteurs ont établi un lien entre ce paramètre et leur comportement sous actions hydrodynamiques.

Migniot [3] indique que la tension de cisaillement critique de début d'érosion dépend des caractéristiques mécaniques des dépôts, notamment de leur rigidité initiale. Il a trouvé la relation suivante en se basant sur un grand nombre des mesures en canal.

$$
\begin{array}{lll}
u_{*_{c}}=0.009 \cdot \tau_{y}^{0.5} & \text { pour } \tau_{y}>3 \mathrm{~Pa} & \text { (vase plastique) } \\
u_{*_{c}}=0.013 \cdot \tau_{y}^{0.25} & \text { pour } \tau_{\mathrm{y}}<3 \mathrm{~Pa} & \text { (vase fluide) }
\end{array}
$$

où $\tau_{\mathrm{y}}$ est la rigidité initiale $\left[\mathrm{Pa}\right.$ ] et $\mathrm{u}_{\mathrm{c}}$ la vitesse de cisaillement critique d'érosion $\left[\mathrm{m} \mathrm{s}^{-1}\right]$.

Une autre grandeur caractérisant les sols cohésifs est la cohésion non drainée $C_{\mathrm{v}}$. Lorsque la cohésion est importante $\left(\mathrm{C}_{\mathrm{u}} \geq \mathrm{IkPa}\right)$ ce paramètre peut être déterminé en utilisant un scissomètre ou un pénétromètre à cône.

Ainsi, Partheniades [4] considère que la tension de cisaillement critique d'érosion des matériaux sous une action hydrodynamique est proportionnelle à la cohésion 
du matériau. Ainsi, le début d'érosion se produirait pour une tension de cisaillement critique $\tau_{\mathrm{n}}$ donnée par l'expression suivante :

$$
\tau_{o \mathrm{c}}=\mathrm{k} \cdot \mathrm{C}_{\mathrm{u}}
$$

où $\mathrm{k}$ est une constante de proportionnalité et $\mathrm{C}_{\mathrm{u}}$ la cohésion non drainée.

\section{Les mesures}

Le comportement mécanique d'une vase correspondant à huit concentrations différentes a été étudié en laboratoire grâce à trois séries de mesures :

a) mesure de la rigidité initiale au rhéomètre,

b) mesure de la cohésion non drainée au densimètre,

c) mesure de l'éventuelle pente critique d'écoulement.

Tous les essais ont été réalisés sur une vase de la Loire prélevée à Trentemoult. Pour chaque essai, on reconstitue un échantillon à une concentration donnée en homogénéisant par malaxage une partie de vase avec de l'eau du robinet.

En vue d'un traitement statistique des résultats, on répète 15 fois les mesures pour chaque concentration en groupant les essais en 3 séries de 5 mesures successives avec chaque appareil avant de passer à l'appareil suivant.

\subsection{Mesure de la rigidité initiale}

Pour mesurer la rigidité initiale de la vase on a utilisé un viscosimètre de type Brookfield semblable à celui utilisé au LCHF. Le principe est de mesurer le moment de torsion nécessaire pour mettre en mouvement un cylindre immergé dans un fluide. Les résultats sont résumés dans le tableau 1. L'écart-type est calculé par la formule classique :

$$
\begin{array}{ll}
\sigma^{2}=\frac{1}{\mathrm{n}} \sum_{i=1}^{n}\left(X_{i}-\bar{X}\right)^{2} & \text { variance } \\
\sigma=\sqrt{\sigma^{2}} & \text { écart-type }
\end{array}
$$

où $\sigma^{2}$ est la variance, $\sigma$ l'écart-type, $n$ le nombre des mesures, $X_{i}$ la ième mesure et $\overline{\mathrm{X}}$ la moyenne des mesures.

La figure 1 montre la rigidité initiale moyenne en fonction de la concentration et en ajoutant $\pm 2 \sigma$ aux valeurs moyennes, les barres d'erreur maximales et minimales dans un intervalle de confiance de $95 \%$. On constate que l'incertitude relative est d'autant plus grande que la rigidité initiale est forte ; ceci semble être lié à la taille du cylindre du rhéomètre qui est $d$ 'autant plus faible que la rigidité initiale est forte. Ainsi, si le cylindre est petit, la rigidité initiale est intégrée sur une faible surface, ce qui confère au rhéomètre une grande sensibilité à la non homogénéité du matériau. 
Sur cette même figure, on observe la loi ajustée par Migniot pour une vase de la Loire. L'écart par rapport à nos mesures ne peut s'expliquer que par la diversité des complexes vase-eau qu'on peut rencontrer en Loire selon le site et la date de prélèvement des échantillons.

\begin{tabular}{|c|c|c|c|c|c|c|c|c|}
\hline & 1 & 2 & 3 & 4 & 8 & 6 & 3 & 8 \\
\hline otresure & 0,480 & 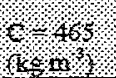 & 8,415 & 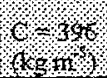 & \% & 4,34 & orv & 6,20 \\
\hline $\mathrm{I}$ & 67.37 & 64.56 & 20.27 & 20.11 & 8.73 & 3.66 & 0.99 & 0.83 \\
\hline 2 & 71.11 & 101.06 & 13.02 & 20.44 & 9.23 & 3.18 & 1.49 & 0.93 \\
\hline 3 & 58.95 & 122.58 & 22.08 & 12.85 & 8.40 & 2.78 & 1.24 & 0.65 \\
\hline 4 & 57.08 & 81.41 & 11.37 & 15.16 & 11.04 & 2.96 & 1.17 & 0.79 \\
\hline 5 & 41.17 & 63.63 & 15.00 & 13.02 & 13.35 & 2.74 & 1.44 & 0.72 \\
\hline 6 & 41.17 & 78.60 & 15.99 & 12.52 & 9.90 & 3.57 & 1.66 & 0.60 \\
\hline 7 & 73.36 & 42.11 & 8.40 & 15.00 & 11.54 & 2.60 & 1.42 & 0.69 \\
\hline 8 & 77.66 & 31.81 & 10.38 & 10.22 & 8.73 & 3.75 & 1.13 & 0.52 \\
\hline 9 & 335.67 & 54.27 & 13.68 & 10.05 & 7.42 & 3.22 & 1.49 & 0.77 \\
\hline 10 & 139.42 & 43.98 & 11.37 & 10.71 & 17.30 & 2.74 & 1.42 & 0.76 \\
\hline 11 & 80.47 & 115.10 & 10.71 & 12.85 & 7.75 & 1.90 & 1.14 & 0.68 \\
\hline 12 & 69.24 & 38.36 & 12.20 & 15.66 & 7.58 & 2.47 & 1.18 & 0.65 \\
\hline 13 & 51.46 & 33.69 & 14.34 & 20.76 & 14.17 & 2.47 & 1.31 & 0.78 \\
\hline 14 & 37.42 & 74.85 & 15.16 & 11.04 & 9.39 & 2.47 & 1.51 & 0.68 \\
\hline 15 & 49.59 & 35.56 & 13.84 & 13.68 & 8.08 & 2.50 & 1.49 & 0.70 \\
\hline movenne & 70,08 & 65,73 & 13,85 & 14,27 & 10,17 & 2.87 & 1,34 & 0.72 \\
\hline ecart type & 29,54 & 27,88 & 3,50 & 3.50 & 2,74 & 0.50 & 0.18 & 0,10 \\
\hline
\end{tabular}

Tableau 1 - Mesures des rigidités initiales en $\mathrm{Pa}$ [2].

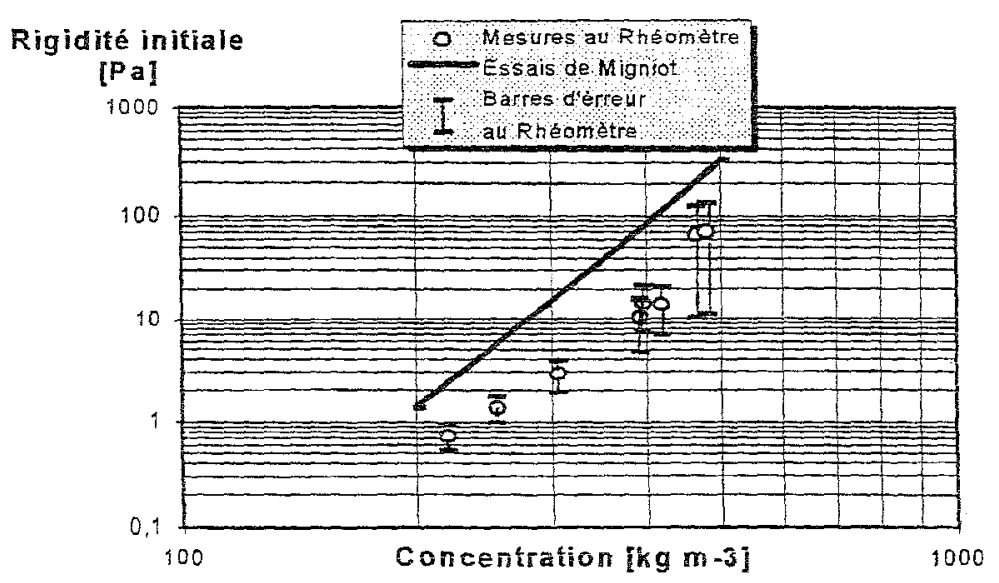

Figure 1 - Variation de la rigidité initiale mesurée à l'aide d'un rhéomètre Brookfield en fonction de la concentration et loi ajustée par Migniot [2].

\subsection{Mesure de la cohésion non drainée au densimètre}

Afin de mesurer des faibles valeurs du seuil de résistance de la vase (laquelle a varié entre $1 \mathrm{~Pa}$ et $250 \mathrm{~Pa}$ dans nos essais), nous avons utilisé un densimètre classique. Ce seuil est évalué en considérant un équilibre des forces. Lorsque le densimètre "flotte" dans une mixture eau-vase de cohésion non nulle, la résistance liée aux frottements sur la surface immergée $\mathrm{A}$ est en équilibre avec le poids déjaugé du densimètre. Nous considérons que la résistance ainsi mesurée 
correspond à la cohésion non drainée $C_{n}$ qui peut être calculée par la formule suivante:

$$
C_{u}=\frac{P-\rho_{\text {sus }} \cdot g \cdot V}{A}
$$

où $\mathrm{Cu}$ est la cohésion non drainée [Pa], $\mathrm{P}$ le poids du densimètre $[N]$, $V$ le volume immergé dans la suspension $\left[\mathrm{m}^{3}\right]$, A la surface en contact avec la vase $\left[\mathrm{m}^{2}\right]$ et $p$ sus la masse volumique de la suspension $\left[\mathrm{kg} \mathrm{m}^{-3}\right]$.

La figure 2 a schématise le densimètre, la figure $2 b$ illustre la mesure de la cohésion en utilisant un densimètre et la figure $2 c$ donne la variation de la surface immergée $\mathrm{A}$ et du volume immergé $\mathrm{V}$ en fonction de la longueur $\mathrm{X}$ plongée dans le mélange eau-vase.

A chaque mesure, le densimètre est introduit doucement dans la vase, afin que les vitesses d'écoulement de la mélange autour du densimètre soient très faibles. Après avoir obtenu un équilibre statique, on mesure la hauteur immergée du densimètre dans la vase. Avec les courbes des caractérșstiques du densimètre, on trouve la surface et le volume immergés.
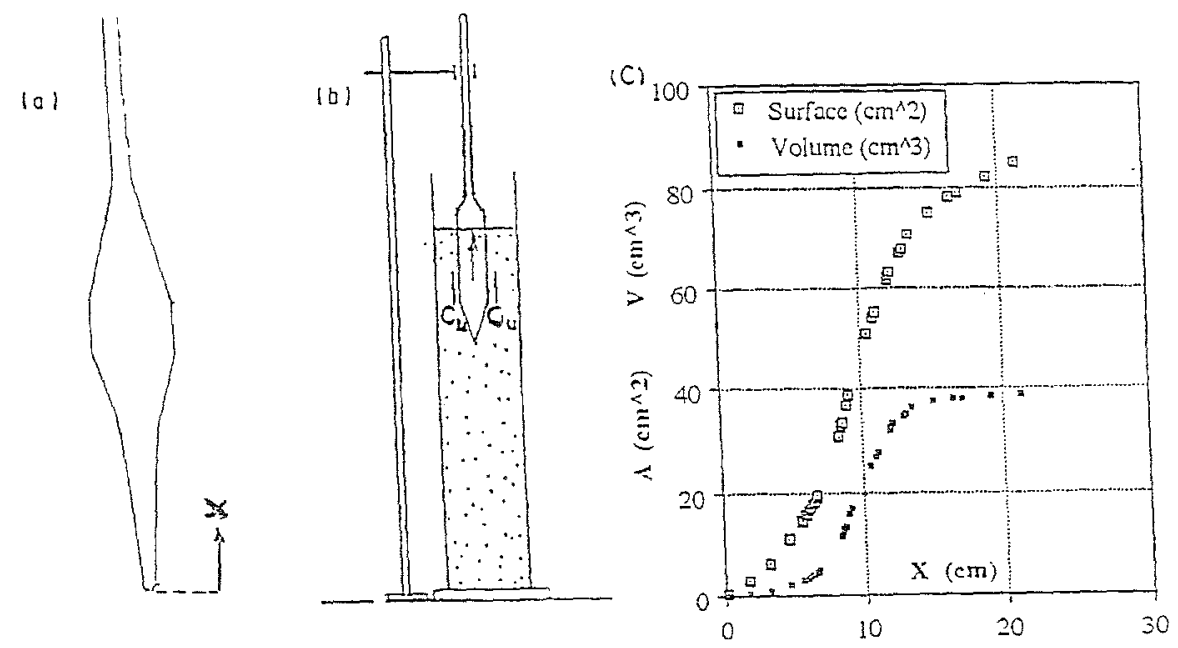

Figure 2 - Mesurer la cohésion à l'aide d'un densimètre.

a) schéma du densimètre.

b) les forces en état équilibre.

c) caractéristiques du densimètre.

Les valeurs de cohésion non drainée obtenues au cours de 8 essais sont résumées dans le tableau 2.

La figure 3 montre la variation de la rigidité initiale mesurée au rhéomètre et la cohésion non drainée évaluée au densimètre en fonction de la concentration. On y observe que cette dernière est systématiquement 3 à 5 fois supérieure à la première. D'une part, on constate que les écarts relatifs liés aux mesures au densimètre sont plus faibles que ceux associés aux mesures au rhéomètre. Ceci 
serait lié, en reprenant un argument avancé précédemment, à une surface de contact avec la vase plus grande pour le densimètre que pour les cylindres du rhéomètre. D'autre part, les écarts observés entre les deux mesures seraient liés à certains aspects théoriques ayant été ignorés telles que l'éventuelie résistance en pointe du densimètre et la surface de frottement résistante qui ne coïnciderait pas nécessairement avec la surface du densimètre.

\begin{tabular}{|c|c|c|c|c|c|c|c|c|}
\hline & It & \%2 & 3 & 4 & 5 & 6 & 7 & 8 \\
\hline 0,0 trstor & 4,180 & 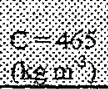 & 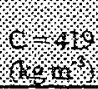 & 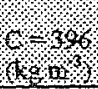 & (1, & 10\% & 0,5 & की \\
\hline 1 & 230.37 & 171.40 & 77.02 & 86.63 & 54.62 & 15.63 & 5.81 & 2.44 \\
\hline 2 & 230.37 & 177.75 & 77.02 & 77.59 & 58.00 & 15.63 & 7.00 & 2.44 \\
\hline 3 & 242.18 & 177.75 & 77.02 & 86.63 & 51.39 & 16.73 & 7.00 & 2.44 \\
\hline 4 & 263.33 & 171.40 & 77.02 & 86.63 & 58.00 & 16.73 & 8,18 & 3.28 \\
\hline 5 & 254.64 & $\$ 77.75$ & 77.02 & 77.59 & 54.62 & 16.73 & 7.00 & 2.87 \\
\hline 6 & 301.46 & 165.13 & 68.51 & 86.63 & 54.62 & 16.73 & 7.00 & 3.28 \\
\hline 7 & 254.64 & 165.13 & 72.66 & 86.63 & 54.62 & 17.82 & 8.18 & 2.44 \\
\hline 8 & 263.33 & 165.13 & 77.02 & 86.63 & 51.39 & 16.73 & 7.00 & 3.28 \\
\hline 9 & 222.83 & 171.40 & 77.02 & 82.06 & 58.00 & 16.73 & 8.18 & 3.28 \\
\hline 10 & 208.53 & 165.13 & 77.02 & 77.59 & 54.62 & 15.63 & 8.18 & 3.28 \\
\hline 11 & 263.33 & 165.13 & 77.02 & 77.59 & 58.00 & 14.51 & 5.81 & 3.28 \\
\hline 12 & 246.26 & 177.75 & 72.66 & 86.63 & 58.00 & 14.31 & 7.00 & 3.28 \\
\hline 13 & 215.56 & 184.15 & 72.66 & 77.59 & 51.39 & 15.63 & 7.00 & 3.28 \\
\hline 14 & 238.17 & 177.75 & 77.02 & 73.24 & 58.00 & 15.63 & 5.81 & 3.28 \\
\hline 15 & 215.56 & 171.40 & 77.02 & 73.24 & 58.00 & 15.63 & 8.18 & 3.28 \\
\hline movenne & 243.37 & 172.28 & 75.58 & 81.53 & 55.55 & 16.07 & 7.15 & 3.03 \\
\hline ecart type & 23.64 & 6.05 & 2.56 & 5.15 & 2.65 & 0.88 & 0.85 & 0.37 \\
\hline
\end{tabular}

Tableau 2. - Mesures des cohésions non drainées en $\mathrm{Pa}$ [2].

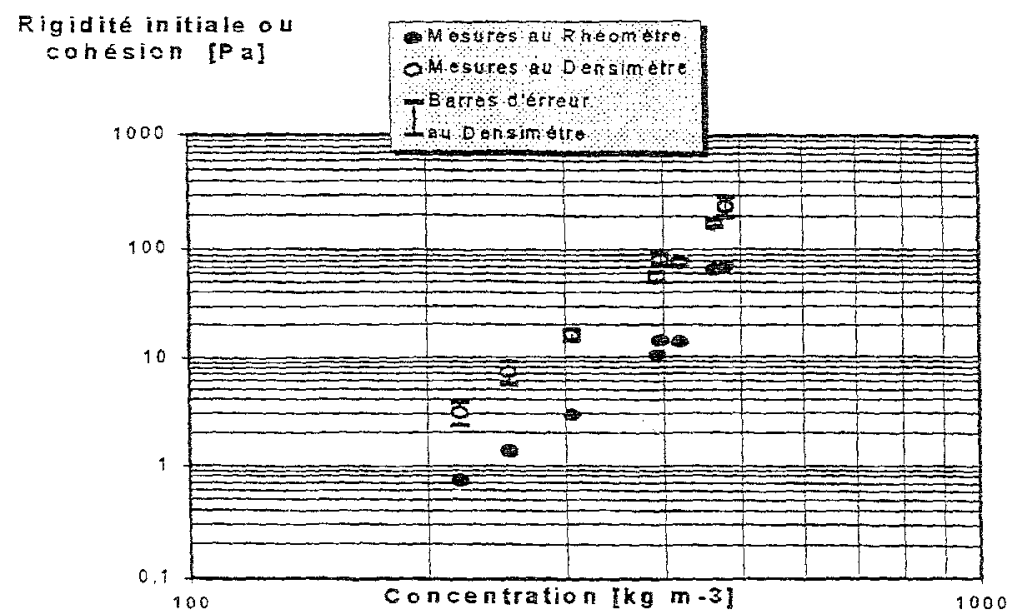

Figure 3 - Variation de la rigidité initiale et de la cohésion non drainée en fonction de la concentration [2]

\subsection{Observation qualitative de la déformation ou de l'écoulement}

Afin de mieux comprendre le comportement des vases on a cherché la pente audessus de laquelle la vase s'écoule. Pour ce faire, on a rempli de vase un cylindre creux ( $2 \mathrm{~mm}$ de la hauteur et $14,5 \mathrm{~mm}$ du diamètre) situé sur une plaque en 
plexiglas. Après avoir enlevé le cylindre on a incliné la plaque doucement jusqu'à ce quili y ait un écoulement observé visuellement.

Ces essais permettent l'observation qualitative du comportement de la vase lié à la tension de cisaillement appliquée. On observe soit un comportement solide lorsquil n'y a pas d'écoulement, soit un comportement liquide dans le cas contraire [1].

La tension de cisaillement moyenne exercée sur la vase à une distance infinitésimale de la plaque peut être calculée en considérant la forme initiale de l'échantillon, comme suit:

$$
\tau=\frac{P \cdot \sin \alpha}{S}
$$

où $\mathrm{P}$ est le poids d'échantillon [N], $\alpha$ l'angle de l'inclinaison et $\mathrm{S}$ la surface initiale d'échantillon en contact à la plaque $\left[\mathrm{m}^{2}\right]$. Les observations sont résumées dans le tableau 3. Pour les concentrations supérieures à $393 \mathrm{~kg} \mathrm{~m}^{-3}$ il y a une déformation mais pas d'écoulement, pour la tension de cisaillement appliquée.

\begin{tabular}{|c|c|c|c|c|c|c|c|c|c|c|}
\hline & 1 & 2 & 3 & 18 & P. & 8 & + & 8 & $\%$ & $4 x$ \\
\hline \% & Xx & X & 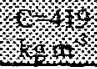 & xyx & ${ }$ x & $x$ & x & $x_{4}$ & \%" & 130 \\
\hline 1 & & \multirow{15}{*}{\multicolumn{4}{|c|}{ Pas d'écoulement }} & 2.50 & 3.34 & 4.73 & $>10$ & $>10$ \\
\hline 2 & & & & & & 2.42 & 3.50 & 5.56 & $>10$ & $>10$ \\
\hline 3 & & & & & & 2.38 & 4.53 & 8.33 & $>10$ & $>10$ \\
\hline 4 & & & & & & 2.50 & 4.19 & 6.06 & $>10$ & $=10$ \\
\hline 5 & & & & & & 2.60 & 4.29 & 5.92 & $=10$ & $=10$ \\
\hline 6 & & & & & & 2.75 & 4.69 & 6.10 & $>10$ & $>10$ \\
\hline 7 & & & & & & 2.56 & 3.73 & 7.24 & $>10$ & $>10$ \\
\hline 8 & & & & & & 2.70 & 5.45 & 7.38 & $>10$ & $>10$ \\
\hline 9 & & & & & & 2.45 & 3.61 & 6.84 & $>10$ & $>10$ \\
\hline 10 & & & & & & 2.70 & 4.60 & 7.09 & $>10$ & $>10$ \\
\hline 11 & & & & & & 2.63 & 3.16 & 7.00 & $>10$ & $>10$ \\
\hline 12 & & & & & & 2.46 & 4.00 & 5.08 & $>10$ & $>10$ \\
\hline 13 & & & & & & 2.64 & 3.49 & 4.65 & $>10$ & $>10$ \\
\hline 14 & & & & & & 2.45 & 4.41 & 6.83 & $>10$ & $>10$ \\
\hline 15 & & & & & & 2.73 & 4.26 & 7.60 & $\geq 10$ & $=10$ \\
\hline $\begin{array}{c}\mathrm{D} \\
\text { moyenne } \\
(\mathrm{Cm})^{*}\end{array}$ & & & & & & 2.59 & 4.06 & 6.43 & $>10$ & $>10$ \\
\hline $\begin{array}{c}\text { Pente } \\
\text { d'écoulem } \\
\text { ent }\end{array}$ & & & & & & $49^{\circ} 24^{\prime}$ & $35^{\circ}$ & $26^{\circ} 34^{\prime}$ & $12^{\circ} 20^{\prime}$ & $7^{\circ} 40^{\prime}$ \\
\hline $\begin{array}{l}\text { tension } \\
\text { (Pa) }\end{array}$ & $>25.3$ & $>25$ & $>24.6$ & $>24.4$ & $>24.3$ & 17.67 & 12.99 & 9.95 & 4.38 & 2.62 \\
\hline
\end{tabular}

la distance moyenne d'écoulement de la vase

Tableau 3. - Résumé des observations qualitatives du comportement des vases [2]

\section{Conclusions}

Les résultats obtenus pour $C_{u}$ mesuré au densimètre sont 3 à 5 fois supérieures aux valeurs de la rigidité initiale mesurée à l'aide d'un rhéomètre Brookfield. Ces écarts pourraient être liés à certains aspects théoriques ayant été ignorés, telles que la résistance en pointe du densimètre et la surface de frottement résistant qui ne coïncide pas nécessairement avec la surface du densimètre. 
A chaque mesure, la surface en contact entre linstrument de mesure et la vase était plus grande avec le densimetre qu'avec le rheomètre. Les mesures effectuées grâce au densimètre concernent les informations d'une plus grande quantité de vase, tandis que celles réalisées au rhéomètre donnent une rigidité initiale plus ponctuelle. De ce fait, afin d'obtenir une moyenne représentative de l'ensemble, il faudrait moins de mesures avec le densimètre (environ 3) qu'avec le rhéomètre (environ 6). Cependant, le théomètre permet d'observer plus clairement la non homogénéité des matériaux. Les résultats obtenus et la comparaison des mesures justifient l'application d'un densimètre type torpille classique, afin d'étudier le comportement des sédiments fins peu cohésifs en place ou en dépôt à terre.

\section{Références Bibliographiques}

[1] Coussot, P. et J.M Piau, 1993, 'L'écoulement des boues', La Recherche, $n^{\circ} 258$, Octobre, Vol. 24, pp 1084-1091.

[2] Hosseini, K., 1995, 'Conditions critiques d'érosion des vases molles', DEA génie civil option génie côtier, Université de Nantes, 47 p.

[3] Migniot, C., 1989, 'Tassement et rhéologie des vases', La Houille Blanche, $\mathrm{n}^{\circ} 2$, pp 95-111.

[4] Partheniades, E., 1984, 'A fondamental framework for cohesive sediment dynamics', Estuarine cohesive sediment dynamics, Edited by A.J. Mehta, Springer - Verlag, pp 219-250

[5] Périgaud, C., 1983. 'Mécanique de l'érosion des vases', La Houille Blanche, $n^{\circ} 7 / 8$, pp $501-512$.

\section{Bibliogranhie}

Mehta, A.J., 1984, 'Characterisation of cohesive sediments properties and transport processes in estuaires', Estuarine cohesive sediment dynamics, Edited by A.J. Mehta, Springer - Verlag, pp 290-325.

Sanchez, M., 1992, 'Modélisation dans un estuaire à marée. Rôle du bouchon vaseux dans la tenue des sols sous-marins', Thèse, déposée et consultable : BU Sciences Nantes, Ecole centrale de Nantes - Université de Nantes, 210 p.

Villaret, C., 1987, 'Etude expérimentale et numérique des lois d'érosion pour des sédiments cohésifs', Thèse, déposée et consultable : BU de Grenoble, $162 \mathrm{p}$. 